\title{
Educación Inclusiva: \\ Tendencias y Perspectivas
}

\author{
Lyda Esperanza Quintero Ayala ${ }^{1}{ }^{(0)}$
}

1.I.E. de Boyacá

lyda.quintero@uptc.edu.co

Como citar: Quintero Ayala, L. (2020).Educación inclusiva:tendencias y perspectivas. EDUCACIÓN Y CIENCIA, (24), e11423. https://doi. org/10.19053/0120-7105.eyc.2020.24. e11423

\section{Resumen}

La educación inclusiva es concebida como un proceso que plantea reconocer y responder a la diversidad de necesidades que presentan los estudiantes para que su aprendizaje sea efectivo, minimizando la exclusión dentro y fuera de las Instituciones Educativas. Este artículo de revisión es producto del estado del arte del proyecto "Prácticas inclusivas: una mirada desde la diversidad", que pretende reconocer las prácticas y los discursos de los docentes de la Institución Educativa de Boyacá en torno a la educación inclusiva. Las investigaciones se clasificaron mediante organización temática de análisis, obteniendo seis apartados que dan cuenta de las perspectivas que se movilizan sobre el objeto de estudio. En cada tendencia, se encontraron hallazgos significativos dirigidos a transformar las prácticas de los docentes. Las categorías establecidas fueron: educación inclusiva a partir del marco legal, actitudes y percepciones de los docentes, redes de apoyo y aprendizaje cooperativo, promoción y buenas prácticas pedagógicas inclusivas, cuestiones sobre la inclusión a propósito de las subjetividades lenguajes, y propuesta curricular en la formación del docente en educación inclusiva.

Palabra clave: educación inclusiva, prácticas inclusivas, diversidad, formación de docentes, diferencia.

Recibido: $12 / 07 / 2020$ | Revisado: 28/07/2020

Aprobado: 23/10/2020 | Publicado: 26/11/2020 


\title{
Inclusive education: trends and perspectives
}

\begin{abstract}
Inclusive education is understood as a process that proposes to recognize and respond to the diversity of needs presented by students so that their learning be able to effective, minimizing exclusion within and outside of Educational Institutions. This review article is the state of the art related to the project "Inclusive practices: a view from diversity", which aims to recognize the practices and discourses of teachers in the Educational Institution of Boyacá about inclusive education. The investigations were classified by thematic organization of analysis, obtaining six sections that show the perspectives that are mobilized on the object of study. In each trend, significant results were found aimed at transforming teachers' practices. The established categories were: inclusive education based on the legal framework, attitudes and perceptions of teachers, support networks and cooperative learning, promotion and alright inclusive pedagogical practices, inclusion issues, in terms of subjectivities - languages, and curricular proposal in the teacher training. in inclusive education.
\end{abstract}

Keywords: inclusive education, inclusive practices, diversity, teacher training, difference.

\section{Introducción}

Este artículo expone a través de un ejercicio de revisión documental, la producción académica sobre inclusión educativa y prácticas pedagógicas desarrolladas por docentes. Estos antecedentes hacen parte del proyecto: Prácticas inclusivas: una mirada desde la diversidad, desarrollado en la Institución Educativa de Boyacá. Es importante resaltar la necesidad de realizar estados del arte, pues según Jiménez (2006), éstos se constituyen en "el punto de partida para generar producciones nuevas e inéditas, que aporten a la investigación y a la comunidad académica" (p. 33).

\section{Ruta Metodológica}

La investigación comprendió una revisión documental que aborda desde diversas perspectivas las prácticas inclusivas de docentes, indagando particularmente los objetivos, alcances, metodologías aplicadas y resultados obtenidos de 55 documentos entre artículos científicos, tesis de maestría y doctorado, y capítulos de libros resultados de investigación. Para ello, se realizó la búsqueda en bases de datos especializadas utilizando los descriptores inclusión educativa y prácticas pedagógicas inclusivas. Posteriormente, se utilizaron fichas temáticas, analíticas y matrices de referencia que permitieron la organización y categorización que se detallará a continuación. 


\section{Educación Inclusiva: Marco Legal}

En esta categoría se precisan políticas y estrategias desarrolladas en Latinoamérica y España en torno a la inclusión educativa, como mecanismo que permite el acceso a la educación de todas las personas.

Desde una perspectiva amplia, Echeita et al. (2009), han realizado estudios en España para reconocer la situación y detectar barreras que interfieren notablemente en el proceso de inclusión de estudiantes con necesidades de apoyo educativo y que limitan la participación y el aprendizaje. Entre estas, se encuentran las concepciones que tienen los agentes educativos sobre la acogida en el proceso de escolarización, los mecanismos de apoyo para atender cada situación particular.

Beltrán, Martínez y Vargas (2015), muestran avances y retos en materia de educación inclusiva en Colombia a través de un análisis comparativo entre las políticas y estrategias desarrolladas a nivel nacional y en España. Concluyen, que se ha avanzado en el reconocimiento de la educación inclusiva como principio en la búsqueda de la educación para todos, pero, aunque el Ministerio de Educación Nacional (MEN) ha atendido orientaciones de la UNESCO, el reto de la educación inclusiva para todos aún está lejos de alcanzarse. Así mismo, destacan que, en Colombia se está transitando de un modelo de integración de los estudiantes con discapacidad a otro de inclusión, lo cual implica que la escuela se transforme y que la gestión escolar se modifique para responder a condiciones particulares.

En Colombia de acuerdo al Decreto 1421 (2017), se han adelantado políticas que reglamenten la atención de la población con discapacidad. Así mismo, esta norma define ajustes razonables como acciones, adaptaciones, estrategias, apoyos, recursos o modificaciones necesarias del sistema educativo y la gestión escolar, basadas en las necesidades específicas de cada estudiante, que contribuyen a los procesos de educación inclusiva, los cuales, se ejecutan tras una rigurosa evaluación de las características del estudiante con discapacidad.

En Uruguay Viera y Zeballos (2014), y en Colombia, Flórez (2016), analizan críticamente algunos documentos nacionales para intentar establecer puntos de encuentro y desencuentro entre el marco político normativo y la práctica escolar, relacionadas con la educación inclusiva. También, discuten y revisan términos vinculados con la educación inclusiva y discapacidad, para, por último, conceptualizar la práctica escolar como componente primordial para el desarrollo de una educación inclusiva. Así, Flórez (2016), expone que en algunas Instituciones Educativas (I.E.). no se desarrollan estrategias de enseñanza que respondan a la diversidad individual, con lo cual, existe un gran reto en llevar a la práctica lo establecido en la ley, ya que se requiere de la integración de toda la comunidad educativa y voluntad de los gobernantes de turno.

En Colombia, de acuerdo con Hernández y Velásquez (2016), aunque se 
expiden normas respecto a la educación inclusiva, se continúan reproduciendo prácticas educativas que son inequitativas, discriminatorias y excluyentes. Así mismo, advierten que se cuenta con gran cantidad de documentos normativos sobre la inclusión educativa, no obstante, estos no están acordes a los contenidos y la realidad escolar y social del país.

\section{Actitudes y Percepciones de los Docentes Frente a la Educación Inclusiva}

En este apartado se abordan diferentes perspectivas del maestro respecto a las actitudes, percepciones y necesidades de los docentes en las I.E., acerca de la educación inclusiva, en los escenarios donde se desarrollan las prácticas pedagógicas.

Inicialmente, Torres y Fernández (2015), resaltan la importancia de comprender y escuchar las actitudes y percepciones de los docentes, citando a Pajares (1992), afirman que "entendemos las percepciones como un concepto que engloba las creencias, opiniones y consideraciones de los protagonistas en sus respectivos contextos de enseñanza-aprendizaje" (p. 6). Para Mora (2019), la actitud "se concibe como un factor que influye en los comportamientos de los docentes y por ende en el desarrollo de sus labores dentro de los procesos de inclusión educativa" (p. 32), con esto las actividades escolares se ven o no favorecidas, lo cual tiene repercusiones en la permanencia o exclusión dentro del entorno escolar. De igual modo, se destacan las dificultades de los maestros que desempeñan procesos de educación inclusiva, pues las condiciones sociales y culturales del contexto, en muchos casos no son favorables.

Así mismo, en el rastreo bibliográfico, los estudios adelantados por autores como Torres y Fernández (2015), Arap (2015), Ruíz (2016), González et al. (2016), Angenscheidt y Navarrete (2017), Montánchez et al. (2017), Peña et al. (2018), Jiménez et al. (2018) y Mora (2019), indagan y resaltan la importancia de conocer las realidades y experiencias de los docentes, quienes a diario se ven enfrentados a situaciones de incertidumbre frente a la educación inclusiva, por tanto, es necesario escuchar sus percepciones, actitudes y necesidades en el ejercicio pedagógico.

Estas investigaciones reúnen información relevante sobre las actitudes, percepciones y necesidades de los docentes de las I.E. Se evidencia que los docentes reconocen el modelo para afrontar las prácticas inclusivas con la finalidad de trabajar en lo que obstaculiza el desarrollo de esta tarea. Los estudios muestran que algunas barreras que impiden la educación inclusiva son la poca sensibilización social y la falta de formación de los docentes. Así mismo, los maestros plantean "la necesidad de formación y a nivel estructural la disminución de alumnos por aulas". (Ruíz, 2016, p. 131). Pues, los primeros involucrados en este proceso han de ser los maestros mediante la formación que se requiere en la atención a la diversidad.

Por su parte, Mora (2019) y Montánchez et al. (2017) corroboran que los docentes 
manifiestan inconformidades hacia los programas de inclusión que propone el gobierno colombiano, afirman que "hay una falta de garantías y recursos para ello y aun así se está llevando a cabo" (Mora, 2019, p. 81). Por ello, es necesario resalar que, la mayoría de I.E. dan cumplimiento con las políticas educativas, sin embargo, esto no es suficiente para garantizar procesos exitosos y de calidad, pues falta voluntad política y garantías para que se adelante un proceso incluyente.

En España la situación es diferente comparada con países de Latinoamérica. Los estudios refieren un análisis de las actitudes y la necesidad manifiesta por los docentes de continuar su formación en cuanto a culturas, políticas y prácticas inclusivas. También, los docentes de este país cuentan con colaboración de grupos interdisciplinarios como tutores, profesores de apoyo, equipos de orientación escolar para la atención a la población, no obstante, Torres y Fernández (2015) advierten que este apoyo se centra en la "Atención individualizada a los alumnos con ANEAE (Alumnos con Necesidades Educativas Especiales y Necesidad Específicas de Apoyo Educativo) fuera del aula ordinaria”(p. 14).

En el nivel de educación superior, Peña et al. (2018) al analizar las percepciones de los futuros docentes de educación básica sobre la educación inclusiva, encontraron que la mayoría de docentes en formación presentan concepciones contradictorias en cuanto al tema, pues consideran que la población con Necesidades Educativas Especiales (NEE) debería acudir a centros especializados para su formación, así mismo, opinan que la escuela solo tiene que atender a cierto grupo poblacional, que la inclusión educativa es una utopía y que los docentes no cuentan con la formación necesaria para atender a la diversidad. Lo anterior, evidencia la necesidad de evaluar el currículo de los programas de formación de docentes, igualmente, permite hacer un llamado urgente a las universidades para revisar los planes de estudio y tomar acciones que propicien la formación de los docentes, según las políticas y adelantos que se han generado en el tema a nivel internacional.

\section{Redes de Apoyo y Aprendizaje Cooperativo}

En esta tendencia, se identificaron algunos estudios que dirigen su interés a estrategias metodológicas aplicadas al aula de clase. Así mismo, se exponen investigaciones donde se logran construir redes de apoyo y colaboración en diferentes centros educativos en Inglaterra y España, los cuales muestran las potencialidades para favorecer la educación inclusiva.

Así, para Moliner et al. (2017), la razón del aprendizaje colaborativo es lograr trabajar juntos para la consecución de objetivos comunes. "Se trata de una situación en la que todos trabajan para obtener resultados beneficiosos para ellos mismos y para los demás miembros del grupo. Los alumnos trabajan en grupos para maximizar su propio aprendizaje y el de los demás” (Moliner et al., 2017, p. 45). Sin embargo, hay que destacar que las actividades del aprendizaje cooperativo exigen 
del docente una mayor preparación y estructura para su ejecución e implica más participación por parte de los estudiantes. El trabajo colaborativo posibilita en los educandos la adquisición de habilidades sociales, comunicativas y de interacción con sus compañeros, generando experiencias de aprendizaje que fortalecen el proceso de educación inclusiva.

Vázquez et al. (2015), sugieren que las prácticas de los docentes aún son tradicionales, y tendientes a homogenizar los procesos y los estudiantes, lo cual, muestra la necesidad de realizar prácticas de inclusión educativa y de aprendizaje colaborativo donde los actores en el proceso sean docentes y estudiantes. Por su parte, García y Hernández (2016) exponen que al utilizar el aprendizaje cooperativo en el aula se generan habilidades para la comprensión de emociones y habilidades académicas, lo que incide positivamente en las habilidades sociales. Además, consideran que el aprendizaje colaborativo "facilita el trabajo con grupos heterogéneos y da cabida a los alumnos con Necesidades Específicas de Apoyo Educativo, atendiendo a la diversidad desde un enfoque inclusivo y permitiendo que todos puedan aprender juntos en el aula ordinaria” (p. 20).

Así mismo, Azorín, (2017) y Arnaiz et al. (2018), desarrollan investigaciones a nivel macro, conformando redes de apoyo y colaboración entre diferentes centros educativos en Inglaterra y España, donde proponen instrumentos que permiten evaluar la atención a la diversidad y la educación inclusiva. Los autores plantean que las redes de colaboración entre I.E. son herramientas para lograr cambios significativos en el contexto escolar. Estos estudios concluyen que "las escuelas no pueden superar las barreras y las desigualdades por sí solas. La inclusión precisa de responsabilidades educativas, políticas y sociales compartidas” (Azorín, 2017, p. 43). Lo anterior deja al descubierto, un panorama complejo en Latinoamérica comparado con otros países, pues, si bien es cierto, las I.E. establecen convenios con universidades, al parecer no existe una efectiva articulación de enfoques y prioridades. Por un lado, las I. E. suman esfuerzos para lograr procesos significativos de enseñanza, y por otro lado, las universidades priorizan las prácticas investigativas, ante esto, es necesario establecer equilibrio entre las dos perspectivas, posibilitando intercambios productivos en beneficio de la comunidad educativa. El rol de la escuela está cambiando y es necesario trascender los espacios de las I.E. a las comunidades, hacer extensa su labor educativa y formadora a otros ambientes circundantes para establecer redes interinstitucionales.

\section{Promoción y Buenas Prácticas Pedagógicas Inclusivas}

En el esquema central de la educación, las prácticas pedagógicas son la parte fundamental que permite la interacción entre docentes y estudiantes, pues son las estrategias que los docentes crean y que favorecen el aprendizaje para todos. En esta 
tendencia, se exponen investigaciones que reflejan diferentes experiencias educativas para promover prácticas pedagógicas que conlleven a la educación inclusiva.

Gómez (2014), interpreta la gestión del equipo directivo de una I.E. enfatizando en la importancia de las actitudes positivas de las directivas frente a la diversidad. Una de las características que promueven los equipos directivos "es ser el dinamizador y promotor de buenas prácticas que den lugar al desarrollo integral de todo el alumnado" (Gómez, 2014, p. 10). Así mismo, se determinó que la convivencia, la colaboración y el liderazgo distribuido son los elementos básicos en la atención a la diversidad. Calderón (2014) resalta la importancia de los roles de los agentes educativos para el buen desarrollo de la inclusión de los niños, y la importancia de contar con un currículo abierto y flexible para realizar los ajustes y cambios de acuerdo a las necesidades de los estudiantes.

Igualmente, Azorín et al. (2017) realizan una revisión de instrumentos dirigidos a la atención de la diversidad y la educación inclusiva, donde categorizan la información según los miembros de la comunidad educativa realizando un análisis crítico de sus temáticas. El análisis de estos instrumentos permite la reflexión de lo que sucede en el contexto de las I.E. Desde otra perspectiva, Balongo y Mérida (2017) mediante actividades inclusivas desarrolladas en un aula de clase infantil, encuentran que a través de la aplicación de proyectos de trabajo (PT) se favorece la creación de contextos inclusivos en las aulas.

De otra parte, Bueira y Ramos (2018), describen algunas herramientas para el análisis de las prácticas inclusivas, con el fin de encontrar cuáles son las características de la cultura educativa y qué tanto responden a los criterios de una cultura incluyente. Así mismo, Romero (2015) realizó una adaptación del INDEX Booth y Ainscow "Guía para la evaluación de la educación inclusiva” a partir del cual encontró que las prácticas correspondían a los criterios de una educación inclusiva.

En tanto, Serrato y García (2014), García et al. (2015), Hernández y García (2017), y Flores et al. (2017), plantean la necesidad de evaluar las prácticas inclusivas de los docentes con el objetivo de proporcionarles herramientas teóricas y metodológicas sobre los procesos educativos. Los cuatro estudios utilizaron el instrumento de la Guía de Evaluación de Prácticas Inclusivas en el Aula (GEPIA), el cual permitió registrar las acciones, políticas y actitudes de los docentes en relación con la inclusión. Los resultados obtenidos muestran que, aunque los maestros poseen altas prácticas inclusivas, existe preocupación y temor al desarrollarlas en el aula de clase. Por su parte, García et al. (2015) comparan la labor de los docentes de escuelas regulares que cuentan con apoyo en educación especial y Centros de Atención Múltiple, y concluyen que en estos centros se cuenta con más experiencias inclusivas respecto a sus pares, esto también se explica por las condiciones de trabajo (número reducido de estudiantes por grupo y el conocimiento que tienen en discapacidad). 
En investigaciones adelantadas por Conrado (2016) y Herrera (2017), sugieren el desarrollo de propuestas pedagógicas para fortalecer los procesos inclusivos. En la escuela se deben generar espacios académicos que fomenten reflexiones pedagógicas en torno a las I.E. y nuevas alternativas de enseñanza. También, se evidencian resultados positivos a partir del trabajo colaborativo de estudiantes y docentes para el reconocimiento de la diversidad (Conrado, 2016).

Desde lo educativo se proponen disposiciones que fortalecen el desarrollo de procesos inclusivos, los cuales han favorecido a la comunidad educativa. Beltrán, Martínez y Torrado (2015), Kremer y Quijano (2015), Figueroa et al. (2017) y Carrillo (2018), evidencian resultados de experiencias que atienden necesidades particulares del contexto, y concluyen que abordar la inclusión no solo hace referencia a la discapacidad, sino que las I.E. deben incluir a la población víctima del conflicto armado, habitantes de frontera, poblaciones afectadas por desastres naturales y poblaciones rurales en condición de pobreza. Así mismo, Figueroa et al. (2017), retoman cuatro categorías: competencia, singularidad, vínculos y participación, las cuales se relacionan con el paradigma de la alteridad, considerado como elementos claves para lograr la inclusión educativa.

Corona et al., (2017), realiza una mirada comparativa entre Chile, Colombia y México, sobre la situación de niños con discapacidad intelectual y en situación de calle, en éste se analiza y describe un caso por cada país, se efectúa una comparación frente a la inclusión educativa y se rescatan prácticas existosas al respecto, argumentando que la educación inclusiva es un término amplio, que no se puede limitar a un solo grupo poblacional. Concluyen haciendo un llamado a los docentes para continuar con procesos de formación permanente que atiendan la diversidad desde una perspectiva amplia.

Por su parte, Ardila y Vásquez (2018) vinculan a estudiantes, padres de familia y docentes, con la finalidad de caracterizar las creencias y los imaginarios que se tienen con respecto a la educación inclusiva, teniendo en cuenta que, en el contexto escolar aumentan los casos de estudiantes con diferentes situaciones de vulnerabilidad, quienes deben ser acogidos, atendidos y formados desde una actitud de respeto, pluralidad e igualdad de oportunidades. De otro lado, Carrillo et al. (2018), se centran en la aplicación de cuestionarios para comparar las prácticas pedagógicas de los docentes, y concluyen, que una continua capacitación a los maestros permite el fortalecimiento de la comunicación, la relación disciplinar y aplicación de estrategias pedagógicas fundamentadas en modelos constructivistas.

Así mismo, Castillo (2015) manifiesta que los docentes están llamados a afrontar grandes retos para desarrollar su práctica pedagógica, por tanto, la formación del maestro debe girar en torno a "los ámbitos pedagógicos, psicológicos e ideológicos para atender la diversidad, construir diferentes escenarios de aprendizaje y garantizar una educación con componentes de educación inclusiva y de calidad” (p. 28). También considera la educación inclusiva como la herramienta que el docente debe 
incorporar en su formación para atender la diversidad. Respecto a los maestros en formación, Zamudio y Martínez (2017) y San Martin, Villalobos, Muñoz y Wyman (2017) señalan la necesidad de que los programas de formación indaguen más sobre un enfoque integrador, utilizando para ello el aprendizaje colaborativo. Estas investigaciones demuestran una preocupación permanente por adquirir herramientas para mejorar las prácticas pedagógicas inclusivas de los docentes, desde donde se brinden estrategias de solución a las diferentes circunstancias y realidades por las que atraviesan los estudiantes, para así, reconocer los discursos en torno a la educación inclusiva y poder transformar esos espacios. Según Leiva (2013) (como se citó en Echeita y Ainscow, 2011) "La inclusión es un proceso, un camino que emprenden las escuelas con el fin último de conseguir progresivamente que todos sus miembros se sientan parte integrante del centro, aceptados y valorados". (párr. 14, sección Educación Inclusiva: fundamentos teóricos). La inclusión requiere que toda la comunidad educativa participe de manera efectiva, para transformar los contextos educativos con igualdad y equidad.

\section{Cuestiones Sobre la Inclusión: Subjetividades - Lenguajes}

Esta tendencia se caracteriza por emprender una mirada hacia la diferencia y la pregunta por la inclusión, en este sentido, Skliar (2007) intenta relacionar la situación con fundamentos, prácticas y realidades de la educación denominada inclusiva. Promueve sus conocimientos hacia una reflexión sobre la inclusión educativa y la inclusión del otro y de todos, en los espacios donde se desarrolla la vida social, defendiendo la posición de la inclusión educativa como derecho humano, no como obligación universal.

A partir de los aportes de Skliar, Patiño y Mariño (2018), muestran cómo las personas que tienen alguna discapacidad han sido señaladas por la misma sociedad; "se sospecha acerca de si el otro puede ser 'tan humano' como lo somos en apariencia, nosotros mismos [...] 'Eso' que usan los sordos ¿̇es una lengua? 'Eso' que hacen los ciegos para leer ¿es lectura?” (p. 40, tomado de Skliar y Téllez, 2008). Tal situación refleja cómo se rotula a esta población como diferente o inferior, ocasionando indiferencia ante sus necesidades. Este planteamiento va más allá, si se pregunta por si el docente está lo suficientemente preparado para la atención a la diversidad, o mejor, si cada maestro está en la capacidad de reconocer qué tan dispuesto está a involucrarse en el mundo de cada estudiante que recibe en su aula de clases, cuál sería la acogida que está decidido a brindar sin esperar nada a cambio; se trata también de ponerse en el lugar del otro.

\section{Propuesta Curricular en la Formación del Docente en Educación Inclusiva}

En este apartado, se identificaron investigaciones que se centran en indagar la relación de currículo y diversidad, y presentan supuestos teóricos sobre la 
formación del profesorado desde una perspectiva organizativa, curricular y de desarrollo profesional, tomando como base el aprendizaje cooperativo y el trabajo por proyectos (Hernández \& Avilés, 2017).

Inicialmente, Torres y Fernández (2015) señalan tres cuestiones a indagar, la primera, relacionada a niños con NEE que reciben "otro tipo de educación”, para determinar si experimentan o no cambios en su proceso. Una segunda, sugiere revisar si la inclusión educativa ha supuesto algunos avances, cambios e innovaciones, o si por el contrario, se ha perpetuado el papel que durante muchos años tuvo la Educación Especial tradicional. Y, en tercer lugar, si la integración ha hecho resonar en el profesorado la idea de alumnos diferentes con necesidades que requieren respuestas particulares. Estos planteamientos insinúan que se continua con la misma educación especial tradicional, quedando en duda si han existido cambios significativos, o por el contrario, solamente han variado las denominaciones.

Por otra parte, Amer y Pascual (2015) centraron su atención en el contexto y en circunstancias sociofamiliares de los estudiantes, pues estos factores están íntimamente relacionados para que los estudiantes abandonen o fracasen en su proceso escolar, debido a las condiciones económicas, la formación y aspiraciones que poseen las familias. Los autores encontraron que estos escenarios afectan al grupo de estudiantes en las I. E. Así mismo en los diferentes centros donde se abordó la investigación se evidenciaron iniciativas de contenidos que tienen en cuenta la atención personalizada, emociones, auto imagen y autoestima de los estudiantes.

Desde otra perspectiva, estudios realizados por San Martín, Salas, Howard \& Blanco (2017), hace referencia a los procesos de diversificación de la enseñanza en escuelas diferenciales en Chile. En Brasil y Ecuador, exploran las percepciones de docentes en relación a la educación inclusiva y sus implicaciones para pensar y analizar el currículo escolar. De Souza et al. (2018) plantea que:

pensar la inclusión educativa es reflexionar sobre la necesidad de un proyecto social y educativo que satisface las necesidades de formación de los seres humanos para que tengan acceso a una parte de la cultura (conocimiento, cultura) y, por lo tanto, aprender conceptos construidos históricamente por la humanidad (p. 341).

Las I. E. tienen el reto de preparar a sus estudiantes a través de un plan de estudios que propicie prácticas inclusivas. Este plan debe estar cimentado bajo un panorama amplio que garantice el derecho a la educación para todos. En este sentido, Pinto (2016) expone que al aplicar las adaptaciones curriculares a través de estrategias metodológicas en estudiantes con NEE asociados a discapacidad, se encontró que los docentes dieron respuestas negativas en niveles bajos, debido a que las modificaciones realizadas en las estrategias metodológicas no fueron 
significativas para sus estudiantes, posiblemente por la apatía que existe sobre el tema de la discapacidad. Esto reafirma la importancia que tiene el cambio de actitud y la disposición de los docentes para que se concreten prácticas inclusivas que favorezcan el aprendizaje de todos y todas.

Por su parte, Poblete (2018) y Mora (2019) buscaron conocer la forma en que los centros educativos incluyen en el currículo la diversidad que representan niños y niñas migrantes, y las adaptaciones que realizan los docentes para asegurar su aprendizaje. Se encontró, que a pesar que las I.E. y los docentes realizan esfuerzos para trabajar con esta población, se hallan dos dificultades: la poca flexibilidad del currículo, y la falta de preparación de los docentes para llevar a cabo las adaptaciones necesarias. Como se aprecia, la diversidad toca muchos de los aspectos sociales por los que atraviesan los niños y niñas, y es en la escuela donde muchos de ellos esperan encontrar el refugio, consuelo y las posibilidades que en su contexto no están presentes.

Finalmente, Acevedo (2017), Corredor (2016) y Silva et al. (2018), buscan comprender cómo acercarse a la inclusión social de las poblaciones diversas en y desde las instituciones de educación superior. Para Acevedo (2017), el curriculo "es un proceso de aprendizaje que debe ser institucionalizado para que se convierta en un proceso vivo, de manera que, a partir de la diversidad, se trasformen las prácticas pedagógicas” (p. 58). En este sentido, el currículo ha de ser adaptado a las necesidades de cada estudiante, lo que implica la formación y capacitación permanente del profesorado en estrategias de planificación educativa para la inclusión, y el trabajo colaborativo de profesionales especialistas y docentes regulares para garantizar un currículo accesible.

\section{Conclusiones}

Esta revisión permitió evidenciar que existen políticas a nivel nacional e internacional encaminadas a la consecución de logros y esfuerzos para consolidar una educación de calidad, que involucre la participación de todos y todas en términos de igualdad y equidad, pero esta a su vez tiene un punto de quiebre, ya que se determinó en la mayoria de investigaciones, que no existe una concordancia entre los contenidos y lo observado en la realidad de los contextos escolares.

Las investigaciones reseñandas, insisten en la importancia de la formación permanente del docente en ejercicio y también en la formación inicial de los docentes para asumir la educación inclusiva. En lo referente a las prácticas pedagógicas inclusivas realizadas por los docentes, se reconoce que es una responsabilidad conjunta, por ello, se requiere aumentar el compromiso de todos los miembros de la comunidad educativa para la obtener mayores beneficios académicos y sociales, y así, propiciar ambientes escolares más inclusivos. Ello exige de los maestros una mayor preparación académica, el desarrollo de habilidades actitudinales, carisma y 
pleno compromiso, pues son elloss los dinamizadores de las actividades y procesos que se generan en las I.E.

La educación inclusiva no se limita solamente a un grupo poblacional como es el caso de la discapacidad, por el contrario, es un término amplio y complejo que aborda un sin número de situaciones con las que conviven los estudiantes, como desigualdad, pobreza, migración y violencia, factores con los que lidia cotidianamente el docente y que repercuten en las aulas de clase.

Así mismo, el estado del arte muestra la importancia de establecer redes de colaboración, apoyo y aprendizaje colaborativo, lo cual se plantea como fundamento para entender que la educación inclusiva es un proceso que requiere entretejer vínculos interdisciplinares, explorar mecanismos como el trabajo colaborativo o el aprendizaje por proyectos, que brinden herramientas para el desarrollo de prácticas efectivas, reconociendo la importancia del aprendizaje social y la flexibilidad curricular en el reconocimiento del el otro, el respeto y la comprensión frente a la diferencia.

\section{Referencias}

Acevedo, S. (2017). Innovación pedagógica y curricular para la inclusión social enla Educación Superior. Revista Pilquen - Sección Psicopedagogía, 14(2), 50-60. http:// revele.uncoma.edu.ar/htdoc/revele/index.php/psico/article/view/1800

Angenscheidt, A., \& Navarrete, I. (2017). Actitudes de los docentes acerca de la educación inclusiva. Ciencias sociológicas, 11(2), 233-243. http://dx.doi. org/10.22235/cp.v11i2.1500

Amer, J., \& Pascual, B. (2015). Las perspectivas del profesorado y el alumnado sobre la implementación de los programas de diversificación curricular en institutos de enseñanza secundaria del municipio de Palma (Mallorca). Revista currículo y formación del profesorado, 19(3), 126-138. https://recyt.fecyt.es/index.php/ profesorado/article/view/43635

Arap, M. (2015). Actitud docente e Inclusión Educativa: Un estudio de caso sobre la Influencia de la Actitud en la práctica docente en clases Inclusivas [Tesis de Maestía, Universidad de la República]. https://hdl.handle.net/20.500.12008/7556

Ardila, C., \& Vásquez, L. K. (2018). Creencias de maestros, padres de familia y niños sobre educación inclusiva en dos instituciones educativas del distrito capital [Tesis de Maestía, Pontificia Universidad Javeriana]. https://repository.javeriana.edu.co/ handle/10554/35283

Arnaiz, S., De Haro, R., \& Azorín, C. (2018). Redes de apoyo y colaboración para la mejora de la educación inclusiva. Revista del currículum y formación del profesorado, 22(2), 29-49. https://recyt.fecyt.es/index.php/profesorado/article/view/66361 
Azorín, C. M. (2017). Redes de colaboración entre escuelas inglesas para la mejora de la inclusion socioeducastiva. Revista del currículo y formación del profesorado, 21(2), 29-48. https://recyt.fecyt.es/index.php/profesorado/article/view/59445

Azorín, C. M., Arnaiz, P., \& Maquilón, J. J. (2017). Revisión de instrumentos sobre atención a la diversidad para una educación inclusiva de calidad. Revista mexicana de investigación educativa, 22(75), 1021-1045. http://www.scielo.org.mx/ scielo.php?script $=$ sci_arttext\&pid=S1405-66662017000401021

Balongo, E., \& Mérida, R. (2017). Proyectos de trabajo: una metodología inclusiva en Educación Infantil. Revista electrónica de investigación educativa, 19(2), 125-142. https://doi.org/10.24320/redie.2017.19.2.1091

Beltrán, Y. I., Martínez, Y. L., \& Torrado, O. E. (2015). Creación de una comunidad de aprendizaje: una experiencia de educación inclusiva en Colombia. Encuentros, 13(2), 57-72. http://dx.doi.org/10.15665/re.v13i2.498

Beltrán, Y. I., Martínez, Y. L., \& Vargas, A. S. (2015). El sistema educativo colombiano en el camino hacia la inclusión. Avances y retos. Educación y Educadores, 18(1), 62-75. http://doi.org/10.5294/edu.2015.18.1.4

Bonilla, R. (2016). Inclusión educativa: una mirada hacia la práctica docente [Tesis de maestría Universidad Pedagógica Nacional]. Repositorio UPN. http://repository. pedagogica.edu.co/handle/20.500.12209/1048

Bueira, C., \& Ramos, R. (2018). Aprendizajes Inclusivos: contribuciones para pensar las prácticas pedagógicas. Appris.

Calderón, M. (2014). La educación inclusiva es nuestra tarea. Educación, 23(40), 4358. http://revistas.pucp.edu.pe/index.php/educacion/article/view/2505

Carrillo, S. (2018). Prácticas Pedagógicas frente a la Educación Inclusiva desde la perspectiva del docente. Revista Espacios, 39(17), 1-18.

Castillo, C. (2015). La educación inclusiva y lineamientos prospectivos de la formación docente: una visión de futuro. Actualidades Investigativas en Educación, 15(2), 1-33. http://dx.doi.org/10.15517/aie.v15i2.

Conrado, K. S. (2016). Diseño e implementación de una propuesta pedagógica para el fortalecimiento de las prácticas inclusivas a partir de los estilos de aprendizaje [Tesis doctoral, Universidad de la Sabana]. https://intellectum.unisabana.edu.co/ handle/10818/22341

Corona, F. Y., Peñaloza, I., \& Vargas, M. L. (2017). La inclusión de niños con discapacidad intelectual y en situación de calle: Una mirada comparativa entre Chile, Colombia y México. Revista Ensayos Pedagógicos, 12(2), 195-215. https://doi. org/10.15359/rep.12-2.9 
Corredor, Z. (2016). Las adecuaciones curriculares como elemento clave para asegurar una educación inclusiva.Educ@ción en Contexto, 2(3), 56-78.

De Souza, J., Rosa, H., \& de Jesus, A. (2018). El currículum y la educación educativa: percepciones de los profesores de educación basica. Roteiro, 43(1), 317 - 344. https://doi.org/10.18593/r.v43i1.14509

Echeita, G., Simón, C., Verdugo, M., A., Sandoval, M., López, M., Calvo I., \& González-Gil, F. (2009). Paradojas y dilemas en el proceso de inclusión educativa en España. Revista de Educación, 153-178. https://repositorio.uam.es/bitstream/ handle/10486/668197/paradojas_echeita_RE_2009.pdf?sequence=1\&isAllowed=y

Figueroa, M. X., Gutiérrez, C., \& Velázquez, Y. (2017). Estrategias de inclusión en contextos escolares. Revista Diversitas - Perspectivas en Psicología, 13(1), 13-26. https://doi.org/10.15332/s1794-9998.2017.0001.01

Flores, V. J., García, I., \& Romero, S. (2017). Prácticas inclusivas en la formación docente en México. Lyberabit, 23(1), 39-56. https://doi.org/10.24265/ liberabit.2017.v23n1.03

Flórez, R. (2016). Educación inclusiva en torno a la discapacidad: encuentros y desencuentros entre marco político y práctica escolar [Tesis de maestría, Universidad Pedagógica Nacional]. Repositorio UPN. http://repository.pedagogica.edu.co/ handle/20.500.12209/1034

García, A. M., \& Hernández, E. (2016). El aprendizaje cooperativo como estrategia para la inclusión del alumnado con tea/as en el aula ordinaria. Revista nacional e internacional de educación inclusiva., 9(2), 18-34. https://revistaeducacioninclusiva. es/index.php/REI/article/view/289 1

García, I., Romero, S., Rubio, S., Flores, V., \& Martínez, A. (2015). Comparación de prácticas inclusivas de docentes de servicios de educación especial y regular en México. Revista Actualidades Investigativas en Educación, 15 (3). http://dx.doi. org/10.15517/AIE.V15I3.20671

Gómez, I. (2014). El Equipo Directivo como Promotor de Buenas Prácticas para la Justicia Social: Hacia un Liderazgo inclusivo. Revista Internacional de Educación para la Justicia Social, 3(2), 141-159. https://revistas.uam.es/riejs/article/view/343

González, F., Martín, E., Poy, R., \& Jenaro, C. (2016). Percepciones del profesorado sobre la inclusión: estudio preliminar. Revista Electrónica Interuniversitaria de Formación del Profesorado, 19(3), 11-24. http://dx.doi.org/10.6018/reifop.19.3.219321

Hernández, B. Y., \& García, I. (2017). Evaluación de actitudes, conocimientos y prácticas de educación inclusiva en docentes de primaria [Ponencia]. XIV Congreso Nacional de Investigación Educativa- COMIE, Universidad Autónoma de San Luis de Potosí. http://www.comie.org.mx/congreso/memoriaelectronica/v14/doc/1382.pdf 
Hernández, C. M., \& Avilés, B. (2017). Propuestas curriculares en la formación docente para la escuela inclusiva. Revista de Estudos Curriculares, 1(8), 87-102. https://www.nonio.uminho.pt/rec/index. php?journal $=$ rec\&page $=$ article $\&$ op $=$ view $\&$ path $\% 5 B \% 5 \mathrm{D}=31 \&$ path $\% 5 \mathrm{~B} \% 5 \mathrm{D}=25$

Hernández, E., \& Velásquez, J. S. (2016). Comprensiones del discurso normativo sobre inclusión educativa en Colombia. El Ágora USB, 16(2), 493-512. https:// doi.org/10.21500/16578031.2447

Herrera, D. G. (2017). Inclusión Educativa desde un Enfoque Sociocultural, apuestas a una Propuesta Pedagógica [Tesis de maestría, Universidad Pedagógica Nacional]. Repositorio UPN. http://repository.pedagogica.edu.co/ handle/20.500.12209/1085

Jiménez, A. (2006). El estado del arte en la investigación en las ciencias sociales. En A. Torres \& A. Jiménez (comp.), La práctica investigativa en ciencias sociales (pp. 2742). Bogotá: Universidad Pedagógica Nacional. http://bibliotecavirtual. clacso.org.ar/ar/libros/colombia/dcsupn/practica.pdf

Jiménez Ruiz, M., Rodríguez Navarro, H., Sánchez Fuentes, S., \& Rodríguez Medina, J. (2018). Construcción del discurso en torno a la Educación Inclusiva. Revista Electrónica Interuniversitaria de Formación del Profesorado, 21(1), 185-217 http:// dx.doi.org/10.6018/reifop.21.1.305771

Kremer, S., \& Quijano O. (2015). Prácticas pedagógicas, promoción de inclusión y diversidad en una institución educativa de Popayán. Plumilla Educativa.15(1), 67-86. https://doi.org/10.30554/plumillaedu.15.833.2015

Leiva, J. J. (2013). De la integración a la inclusión: evolución y cambio en la mentalidad del alumnado universitario de educación especial en un contexto Universitario Español. Revista Actualidades Investigativas en Educación, 13(3), 1-27. http://doi.org/10.15517/AIE.V13I3.12027

Ministerio de Educación Nacional. (2017, 29 de agosto). Decreto 1421. Por el cual se reglamente en el marco de la educación inclusiva la atención educativa a la población con discapacidad. http://es.presidencia.gov.co/normativa/normativa/DECRETO\%20 1421\%20DEL\%2029\%20DE\%20AGOSTO\%20DE\%202017.pdf

Moliner, O., Sanahuja, A., \& Benet, A. (2017). Prácticas Inclusivas en el aula desde la investigación acción. Universitat Jaume I. http://dx.doi.org/10.6035/Sapientia127

Montánchez, M., Carrillo, S., \& Barrera, E., C. S. (2017). Inclusión Educativa: diversidad a partir de la otredad. En M. Graterol, M. Mendoza, J. Contretras, R. Graterol, \& J. Espinosa (Comps.), La base de la pirámide y la innovación frugal en América Latina (pp. 271-282). Universidad de Zulia. https://bonga.unisimon. edu.co/handle/20.500.12442/2103 
Mora, P. A. (2019). Actitudes y prácticas pedagógicas inclusivas [Tesis de Maestría, Universidad Pedagógica Nacional]. Repositorio UPN. https://repository.cinde. org.co/handle/20.500.11907/2443

Patiño, O., \& Mariño, L. D. (2018). Discapacidad e infancia: una historia de los malentendidos. En Ó. Pulido \& M. T. Suárez (Eds.), Infancia, Diversidady Filosofía: Voces, Gritos y Reclamos (pp. 36-70). Editorial UPTC.

Peña, G. X., Peñaloza, W. L., \& Carrillo, M. J. (2018). La educación inclusiva en el proceso de formación docente. Conrado, 14(65), 194-200. https://conrado.ucf. edu.cu/index.php/conrado/article/view/835

Pinto, A. (2016). Adaptación curricular inclusiva en estudiantes que presentan necesidades educativas especiales asociadas a la discapacidad: estrategias metodológicas [Tesis de Maestría. Universidad Tecnológica Equinoccial]. http://repositorio.ute.edu.ec/ handle/123456789/17089

Poblete, R. (2018). El trabajo con la diversidad desde el currículo en escuelas con presencia de niños y niñas migrantes: estudio de casos en escuelas de Santiago de Chile. Perfiles educativos, 40(159), 51-65. https://doi.org/10.22201/ iisue.24486167e.2018.159.58202

Romero, G. (2015). La inclusión educativa en el Colegio República de Bolivia [Tesis de maestría, Pontificia Universidad Javeriana]. https://repository.javeriana.edu.co/ handle/10554/15891

Ruíz, P. (2016). Percepciones de Docentes y Padres sobre la Educación Inclusiva y las Barreras para su Implementación en Lima, Perú. Revista latinoamericana de educación inclusiva, 10(2), 115-133. http://dx.doi.org/10.4067/S0718-

San Martín, C., Salas, N., Howard, S., \& Blanco, P. (2017). Acceso al Currículum Nacional para Todos: Oportunidades y Desafíos de los Procesos de Diversificación de la Enseñanza en Escuelas Diferenciales Chilenas. Revista Latinoamericana de Educación Inclusiva, 11(2). http://dx.doi.org/10.4067/S0718-73782017000200012

San Martin, C., Villalobos, C., Muñoz, C., \& Wyman, I.(2017). Formación inicial docente para la Educación Inclusiva. Análisis de tres programas chilenos de pedagogía en Educación Básica que incorporan la perspectiva de la educación inclusiva. Calidad en la educación, 46, http://dx.doi.org/10.4067/S0718-4565201700010002

Serrato, L., \& García, I. (2014). Evaluación de un programa de intervención para promover prácticas docentes inclusivas. Revista Actualidades Investigativas en Educación, 14(3) 1-25. http://dx.doi.org/10.15517/AIE.V14I3.16093

Silva, M., Llaña M., Maldonado, F., \& Baeza, A. (2018). Algunos desafíos curriculares de la formación ciudadana y la diversidad en Chile. Educación, 27(53), 155-173. https://doi.org/10.18800/educacion.201802.009 
Skliar, C. (2007). La pretensión de la Diversidad o la Diversidad pretensiosa [Panel]. I Jornadas Nacionales de Investigación Educativa. Universidad Nacional de Cuyo. https://feeye.uncuyo.edu.ar/web/posjornadasinve/Pretension_diversidad_o_ Diversidad_pretenciosa.pdf

Torres, J. A., \& Fernández, J. M. (2015). Promoviendo escuelas inclusivas: análisis de las percepciones y necesidades del profesorado desde una perspectiva organizativa, curricular y de desarrollo profesional. Revista Electrónica Interuniversitaria de Formación del Profesorado, 18(1), 177-200. https://doi.org/10.6018/reifop.18.1.214391

Vázquez, M., Méndez, J., \& Mendoza, F. (2015). Educación inclusiva y aprendizaje colaborativo en el aula: un estudio de la práctica docente universitaria. Revista nacional e internacional de educación inclusiva, 8(3), 171-187 https:// revistaeducacioninclusiva.es/index.php/REI/article/view/97

Vieira, A., \& Zeballos, Y. (2014). Inclusión educativa en Uruguay: una revisión posible. Revista Psicología, Conocimiento y Sociedad, 4(2), 237-260 https://revista. psico.edu.uy/index.php/revpsicologia/article/view/243

Zamudio, L. Y., \& Martínez, I. C. (2017). Hacia el fortalecimiento de la atención a la diversidad en Colombia: la educación inclusiva desde la formación de docentes. Paideia, (60), 41-63 https://revistasacademicas.udec.cl/index.php/ paideia/article/view/700 